\title{
EL TRATAMIENTO DE SEMILLAS DE MAÍZ CON MICRONUTRIENTES AUMENTA EL RENDIMIENTO DE GRANO ${ }^{1}$
}

\author{
DURVAL DOURADO NETO ${ }^{2}$, THOMAS NEWTON MARTIN ${ }^{3 *}$, PAULO SERGIO PAVINATO ${ }^{4}$, UBIRAJARA \\ RUSSI NUNES ${ }^{5}$, OTÁVIO DOS SANTOS ESCOBAR ${ }^{6}$, GLAUBER MONÇON FIPKE $^{7}$
}

\begin{abstract}
RESUMEN - El presente experimento fue verificar las respuestas a la aplicación de micronutrientes por la aplicación de tratamiento de semillas y regulador del crecimiento vegetal. El diseño experimental fue de bloques al azar con nueve tratamientos y cuatro repeticiones. Los tratamientos fueron: 1) Testigo: semillas sólido (TS) con $3 \%$ de $\mathrm{P}_{2} \mathrm{O}_{5}, 2 \% \mathrm{~K}_{2} \mathrm{O}, 0,17 \% \mathrm{Cu}, 2,8 \% \mathrm{Mn}$ y $6,2 \% \mathrm{Zn}$ a una dosis de $100 \mathrm{~g} \mathrm{ha}^{-1}$; 2) TS a una dosis de $150 \mathrm{~g} \mathrm{ha}^{-1}$; 3) TS a una dosis de $200 \mathrm{~g} \mathrm{ha}^{-1}$; 4) semillas líquido (TL) con $2 \%$ de $\mathrm{P}_{2} \mathrm{O}_{5}, 1,5 \%$ de $\mathrm{K}_{2} \mathrm{O}, 0,07 \%$ de $\mathrm{Cu}, 1,2 \%$ de Mn y 2,7 \% de Zn a una dosis de $400 \mathrm{~mL} \mathrm{ha}^{-1}$; 5) TL, dosis de $500 \mathrm{ml} \mathrm{ha}^{-1}$, TL a una dosis de $600 \mathrm{~mL} \mathrm{ha}^{-1}$; 6) regulador del crecimiento vegetal que tiene citoquinina + indol-butilo + ácido giberélico, posee clase toxicológica II, $0,09 \mathrm{~g} \mathrm{~L}^{-1}$ citoquinina $0,05 \mathrm{~g}$ de alcohol L $\mathrm{L}^{-1}$ indol-butilo y $0,05 \mathrm{~g} \mathrm{~L}^{-1}$ de ácido giberélico, en una dosis de $500 \mathrm{~mL} \mathrm{ha}^{-1}$; 7) un formulado NPK $(08-28-16) 300 \mathrm{~kg} \mathrm{ha}^{-1}$. El tratamiento de semillas, ya sea en forma sólida o líquida, promueve un aumento en la acumulación de materia seca en la parte aérea y raíces, así como en el número de granos y en el rendimiento de granos de maíz, siendo la dosis de $600 \mathrm{~mL} \mathrm{ha}^{-1}$ de la que promueve las mejores productividades.
\end{abstract}

Palabras clave: Cobre. Manganeso. Zea mays L.. Zinc.

\section{TRATAMENTO DE SEMENTES DE MILHO COM MICRONUTRIENTES AUMENTA A PRODUTIVIDADE DE GRÃOS}

\begin{abstract}
RESUMO - O presente experimento teve como objetivo verificar as respostas da aplicação de micronutrientes com reguladores vegetais em tratamento de sementes. $\mathrm{O}$ delineamento experimental foi em blocos ao acaso com nove tratamentos e quatro repetições. Os tratamentos foram: 1) Testemunha: sementes sólidas (TS) com $3 \%$ de $\mathrm{P}_{2} \mathrm{O}_{5}, 2 \% \mathrm{~K}_{2} \mathrm{O}, 0,17 \% \mathrm{Cu}, 2,8 \% \mathrm{Mn}$ e $6,2 \% \mathrm{Zn}$ e uma dose de $100 \mathrm{~g} \mathrm{ha}^{-1}$; 2) TS com uma dose de $150 \mathrm{~g} \mathrm{ha}^{-1}$; 3) TS com uma dose de $200 \mathrm{~g} \mathrm{ha}^{-1}$; 4) Sementes líquidas (TL) com $2 \%$ de $\mathrm{P}_{2} \mathrm{O}_{5}, 1,5 \%$ de $\mathrm{K}_{2} \mathrm{O}$, $0,07 \%$ de $\mathrm{Cu}, 1,2 \%$ de $\mathrm{Mn}$ e $2,7 \%$ de $\mathrm{Zn}$ a uma dose de $400 \mathrm{~mL} \mathrm{ha}^{-1}$; 5) TL, a dose de $500 \mathrm{~mL} \mathrm{ha}^{-1}$, TL com uma dose de $600 \mathrm{~mL} \mathrm{ha}^{-1}$; 6) regulador de crescimento vegetal que continha citocinina + indol-butilo + ácido giberélico, com classe toxicológica II, $0,09 \mathrm{~g} \mathrm{~L}^{-1}$ citocinina $0,05 \mathrm{~g}_{\text {de álcool L}}{ }^{-1}$ indol-butilo e $0,05 \mathrm{~g} \mathrm{~L}^{-1} \mathrm{de}$ ácido giberélico, em uma dose de $500 \mathrm{~mL} \mathrm{ha}^{-1}$; 7) um formulado de NPK $(08-28-16) 300 \mathrm{~kg} \mathrm{ha}^{-1}$. O tratamento composto por aplicação líquida em sementes, $600 \mathrm{~mL} \mathrm{ha}^{-1}$, apresentou a maiorers massa seca de parte aérea e radicular, número de grãos e produtividade de grãos.
\end{abstract}

Palavras-chave: Cobre. Manganês. Zea mays L.. Zinco.

\footnotetext{
*Autor correspondiente

${ }^{1}$ Recibido el 26/08/2014; Aceptado 06/04/2015.

${ }^{2}$ ESALQ/USP, 13418-900, Piracicaba-SP.

${ }^{3}$ Departamento de Fitotecnia, UFSM, 97105-900, Santa Maria-RS; martin.ufsm@gmail.com.

${ }^{4}$ Departamento de Solos, ESALQ/USP, Caixa Postal 11, 13418-260, Piracicaba-SP; pavinato@usp.br.

${ }^{5}$ Departamento de Fitotecnia, UFSM, 97105-900, Santa Maria-RS; russinunes@yahoo.com.br.

${ }^{6}$ Departamento de Fitotecnia, UFSM, 97105-900, Santa Maria-RS; escobar-br@hotmail.com.

${ }^{7}$ Programa de Pós Graduação em Agronomia, UFSM, 97105-900, Santa Maria-RS; gm.fipke@hotmail.com.
} 


\section{INTRODUCCIÓN}

En los últimos años produjo un aumento en la producción de maíz debido al progreso genético y el manejo de la fertilidad del suelo. En Brasil la producción promedio de maíz fue 32.224,5 mil toneladas en 2013/2014 con promedia de $4813 \mathrm{~kg} \mathrm{ha}^{-1}$ (CONAB, 2014). Pero la productividad brasileña se sigue baja, debido a un manejo inadecuado del cultivo, intemperies climáticas y bajo nivel tecnológico adoptado (MARTIN et al., 2011).

Uno de los principales factores que limitan el potencial productivo es la fertilización; frecuentemente se descuidan las dosis y los nutrientes que deben ser aplicados, y cuando se aplican, no se siguen las recomendaciones técnicas para una mejor eficiencia, no permitiendo que el cultivo logre altas tasas de producción (AMARAL FILHO et al., 2005, CRUZ et al., 2008).

Por lo tanto, el área de producción se expandió recientemente para el cerrado brasileño, pero las características de deficiencia de nutrientes de estos suelos, así como la elevada tasa de exportación y el uso de fertilizante NPK de alta concentración, con ausencia de micronutrientes en estos productos (LOPES, 1999, ERNANI et al., 2002), hacen que el uso de micronutrientes por fuentes alternativas, tenga mayor importancia en la productividad del cultivo.

Según Fancelli y Dourado Neto (2000), la cantidad de micronutrientes exportados por el cultivo del maíz para la producción de nueve toneladas de grano, son de $340 \mathrm{~g}$ de manganeso, $110 \mathrm{~g}$ de cobre $\mathrm{y}$ $400 \mathrm{~g}$ de zinc. Otros minerales importantes, principalmente por la exportación de estos elementos por los granos, siendo éstos en $\mathrm{mg} / 100 \mathrm{~g}$ de granos: 287 potasio, 35 sodio, 7 calcio, 210 fósforo, 127 magnesio, 271 hierro, 2,02 zinc, 0,31 cobre, 0,46 manganes y 0,004 selenio (SAULNIER, 2012).

La disponibilidad de micronutrientes en el suelo se verá afectada por factores tales como la textura, la materia orgánica y, principalmente, el $\mathrm{pH}$ del suelo (LEITE et al., 2003). El aumento del pH reduce la disponibilidad de cobre, manganeso y zinc (CAIRES; FONSECA, 2000) debido a la competición de estos nutrientes con los grupos de hidroxilo liberados por el encalado. De acuerdo con Yamada (2004) el uso de micronutrientes en el maíz viene aumentando y la principal formulación utilizada consta de 3,5\% de $\mathrm{Zn}$ y 3,5\% de $\mathrm{Mn}$. El aumento en el uso de micronutrientes se debe a la baja concentración disponible en algunos tipos de suelo y también para controlar algunas enfermedades (TOMAZELA et al., 2006), porque como el $\mathrm{Cu}$ y $\mathrm{Mn}$ participan en los mecanismos de defensa, pueden conferir diferentes niveles de resistencia a las plantas. Autores como Hassegawa et al. (2008) indican que la adición de zinc resultó en un aumento en la frecuencia relativa de Fusarium verticillioides, por otra parte, los auto- res señalan que se debe utilizar de forma equilibrada macronutrientes y micronutrientes, con el fin de, no sólo aumentar la producción de granos, sino como una medida de control de los contaminantes naturales, tales como hongos y toxinas.

La concentración de un determinado nutriente puede indicar el estado nutricional de la planta, ya que existe una relación básica entre esta concentración y el crecimiento o rendimiento del cultivo (BATTAGLIA, 1991). Por lo tanto, el objetivo del presente trabajo, fue verificar las respuestas a la aplicación de micronutrientes por la aplicación de tratamiento de semillas y regulador del crecimiento vegetal.

\section{MATERIALES Y MÉTODOS}

El experimento se llevó a cabo en el año 2009 en el área experimental de la Escola Superior de Agricultura "Luiz de Queiroz", en Piracicaba, SP,

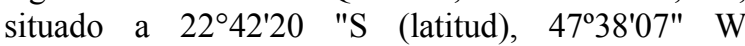
(longitud) y $546 \mathrm{~m}$ (altitud), con una topografía plana. El clima de la región es del tipo Cwa es decir subtropical húmedo, con invierno seco y verano lluvioso, según la clasificación climática de Koppen. El suelo se clasifica como Nitossolo Vermelho Eutroférrico, de textura arcillosa (EMBRAPA, 2013).

El genotipo de maíz que se utilizó fue el 30F80Y, híbrido simple de la Pioneer, que posee gen YieldGard $^{\circledR}$ que confiere resistencia a Spodoptera frugiperda. La siembra fue realizada el 28 de abril de 2009 , con una densidad de 60000 plantas ha $^{-1}$, fueron utilizadas parcelas compuestas por seis filas separadas $0,80 \mathrm{~m}$, con $7 \mathrm{~m}$ de longitud. El diseño experimental utilizado fue bloques al azar con nueve tratamientos y cuatro repeticiones.

Los tratamientos consistieron en aplicaciones de macro y micronutrientes en el momento de la siembra del maíz, los cuales fueron: control (1); tratamiento de semillas sólido (TS - conteniendo: 3\% de $\mathrm{P}_{2} \mathrm{O}_{5}, 2 \%$ de $\mathrm{K}_{2} \mathrm{O}, 0,17 \%$ de $\mathrm{Cu}, 2,8 \%$ de $\mathrm{Mn}$ y $6,2 \%$ de $\mathrm{Zn}$ a una dosis de $\left.100 \mathrm{~g} \mathrm{ha}^{-1}\right)(2) ; 150 \mathrm{~g} \mathrm{ha}^{-1}$ (3), y $200 \mathrm{~g} \mathrm{ha}^{-1}(4)$; tratamiento de semillas líquido (TL - conteniendo $2 \%$ de $\mathrm{P}_{2} \mathrm{O}_{5}, 1,5 \%$ de $\mathrm{K}_{2} \mathrm{O}, 0,07 \%$ de $\mathrm{Cu}, 1,2 \%$ de $\mathrm{Mn}$ y $2,7 \%$ de $\mathrm{Zn}$ a una dosis de $400 \mathrm{~mL} \mathrm{ha}^{-1}(5) ; 500 \mathrm{~mL} \mathrm{~h}^{-1}(6), 600 \mathrm{~mL} \mathrm{~h}^{-1}$ (7); regulador de crecimiento vegetal (8) que tiene citoquinina + indol-butilo + ácido giberélico pose clase toxicológica II, $0,09 \mathrm{~g} \mathrm{~L}^{-1}$ citoquinina $0,05 \mathrm{~g}$ de alcohol $\mathrm{L}^{-1}$ indol-butilo y $0,05 \mathrm{~g} \mathrm{~L}^{-1}$ de ácido giberélico en una dosis de $500 \mathrm{ml} \mathrm{de} \mathrm{ha-1}$ y un formulado NPK (08-28-16) a una dosis más de $300 \mathrm{~kg} \mathrm{ha}^{-1}$ allá del recomendación.

La fertilización de base se aplicó en todos los tratamientos (incluyendo el control), con el formulado NPK (28-08-16), a una dosis de $300 \mathrm{~kg} \mathrm{ha}^{-1}$, basándose en el informe de análisis de suelo (Tabla 1) 
y según las clases propuestas por Raij et al. (1997) para el rendimiento esperado 6-8 $\mathrm{t} \mathrm{ha}^{-1}$. En el día 29 de mayo se llevó a cabo la aplicación de fertilizantes nitrogenados en cobertura, con sulfato de amonio en una dosis de $200 \mathrm{~kg} \mathrm{ha}^{-1}$, cuando las plantas tenían 5 a 6 hojas totalmente desarrolladas.

Tabla 1. Resultados del análisis de química del suelo, tamaño de partícula y micronutrientes (Piracicaba, SP, 2009).

\begin{tabular}{|c|c|c|c|c|c|c|c|c|c|c|c|c|}
\hline \multirow{2}{*}{$\begin{array}{c}\mathrm{pH} \\
\mathrm{CaCl}_{2}\end{array}$} & \multirow{2}{*}{$\frac{\mathrm{MO}}{\mathrm{g} \mathrm{dm}^{-3}}$} & \multirow{2}{*}{$\frac{\mathrm{P}}{\mathrm{mg} \mathrm{dm}^{-3}}$} & \multirow[t]{2}{*}{$\mathrm{K}$} & \multirow[t]{2}{*}{$\mathrm{Ca}$} & $\mathrm{Mg}$ & $\mathrm{Al}$ & $\mathrm{H}+\mathrm{Al}$ & \multirow[t]{2}{*}{ SB } & \multirow[t]{2}{*}{ CTC } & \multirow{2}{*}{$\begin{array}{l}\mathrm{V} \\
\%\end{array}$} & \multirow{2}{*}{$\begin{array}{c}\text { Sat. } \mathrm{Al}^{+3} \\
\mathrm{~m} \%\end{array}$} & \multirow{2}{*}{$\frac{\mathrm{S}-\mathrm{SO}_{4}}{\mathrm{mg} \mathrm{dm}^{-3}}$} \\
\hline & & & & & $--m m$ & $\mathrm{l}_{\mathrm{c}} \mathrm{dm}$ & -------- & & & & & \\
\hline 5,6 & 27 & 15,6 & 1,2 & 88 & 32 & 0 & 25 & 121 & 146 & 83 & 0 & 31 \\
\hline Arcilla & $\begin{array}{l}\text { Limo } \\
---\% \text {--- }\end{array}$ & Arena & Cla & textu & & & $\mathrm{Cu}$ & $\mathrm{Fe}$ & $\begin{array}{c}\mathrm{Zn} \\
-\mathrm{dm}^{-3}\end{array}$ & $\mathrm{Mn}$ & B & \\
\hline 48 & 27 & 25 & $\mathrm{Me}$ & cillo & & & 5,4 & 102 & 7,8 & 32,4 & 0,26 & \\
\hline
\end{tabular}

A los 32 días después de la siembra (V3-V4) fue aplicado los insecticidas Imidacloprido + Tiodicarbe $\left(150 \mathrm{~g} \mathrm{~L}^{-1}+450 \mathrm{~g} \mathrm{~L}^{-1}\right)\left(\right.$ Cropstar $\left.^{\circledR}\right)$ a una dosis de $0,3 \mathrm{~L} \mathrm{ha}^{-1}$ y Metomil $\left(215 \mathrm{~g} \mathrm{~L}^{-1}\right)$ a una dosis de $0,6 \mathrm{~L} \mathrm{ha}^{-1}$. El control de malezas se llevó a cabo a los 28 días después de la siembra, a través de la aplicación de herbicidas Atrazina $\left(500 \mathrm{~g} \mathrm{~L}^{-1}\right)$ (Nortox Atrazina $\left.500 \mathrm{SC}^{\circledR}\right)+$ Tembotriona $\left(420 \mathrm{~g} \mathrm{~L}^{-1}\right)$ (Soberan $400{ }^{\circledR}$ ) a una dosis de $3 \mathrm{~L} \mathrm{ha}^{-1}$ y $0,24 \mathrm{~L} \mathrm{~h}^{-1}$, respectivamente. Las condiciones climáticas en el momento de la aplicación fueron $29^{\circ} \mathrm{C}$ de temperatura ambiente, humedad relativa del aire $46 \%$ y sin viento. El experimento fue regado periódicamente por aspersores mediante un pivote central, con el fin de satisfacer las demandas de agua de las plantas (5-9 $\mathrm{mm}$ de riego al día, según datos de la evapotranspiración local) en los períodos más críticos del cultivo, que involucran la floración y llenado de grano.

A los 27 y 59 días después de la emergencia del maíz (V3 y V8) se recogieron parte aérea y sistema radicular de tres plantas al azar en cada parcela. Estas fracciones de plantas fueron secadas en estufa a $70^{\circ} \mathrm{C}$ durante 72 horas o hasta masa constante, para su posterior pesaje y la determinación de la materia seca de la parte aérea (SDM) y del sistema radicular (MSR).

La cosecha de las parcelas se llevó a cabo el 6 de noviembre de 2009, 181 días después de la emergencia. Los caracteres evaluados en este momento fueron: número de granos por espiga (ESN), número de hileras de granos por espiga (NFE), el diámetro de las espigas (DE), longitud de las espigas (CE), la masa de mil granos (MMG), la productividad de granos (PG) y el porcentaje de aumento de la producción de granos con relación al control (\% A). Para la evaluación del número de granos e hileras por espiga, diámetro y la longitud de las espigas, se tomaron muestras al azar de 10 espigas de cada parcela. Para los demás parámetros fue considerada el área útil de cada parcela.

Los supuestos del modelo matemático fueron probados con pruebas Lilliefors, Bartlett, no aditividad de Tukey y la independencia de los errores. Para el análisis de varianza, los datos de materia seca de la raíz (MSR) fueron transformados en $\sqrt{x+0,5}$, y los demás datos en $\sqrt{x}$. Para las variables que presentaron ser significativas en los promedios fueron compradas mediante al 5\% de probabilidad de error pelo teste de Duncan.

\section{REULTADOS Y DISCUSIÓN}

La materia seca de la parte aérea (MSPA) del maíz a los 27 días después de la emergencia, generalmente, aumenta para todos los tratamientos aplicados con relación al control, se destaca la aplicación del tratamiento de semillas líquido (TL) en dosis de 500 $\left(40,68\right.$ g.ha $\left.^{-1}\right)$ y $600 \mathrm{~mL} \mathrm{ha}{ }^{-1}\left(42,20\right.$ g.ha $\left.{ }^{-1}\right)$. A los 59 días, la aplicación de TL $600 \mathrm{~mL} \mathrm{ha}^{-1}$ fue el que presentó mayor MSPA, no difiere de demás tratamientos con este mismo producto en forma sólida, pero todos se destacaron del tratamiento de control. Para la materia seca de raíces (MSR) a los 27 días, la tendencia fue la misma de la MSPA en este mismo período. Sin embargo, después de 59 días la gran mayoría de los tratamientos proporcionó promedios superiores al control y al TS $100 \mathrm{~mL} \mathrm{ha}^{-1}$ del tratamiento de semillas (Tabla 2). La variación porcentual del aumento en la producción de granos es más del $60 \%$, alcanzando el $160 \%$ de mejores tratamientos.

En general, los resultados de MSPA y MSR fueron influenciados positivamente con la aplicación de los productos a través de los tratamientos de semillas (del segundo al séptimo tratamiento), regulador de crecimiento y la fertilización con NPK. Sin embargo, es importante hacer hincapié en que pequeñas dosis de tratamiento de semillas fueron similares o hasta superiores al NPK, caracterizando una eficiencia importante de este producto en el aprovechamiento por el cultivo. Estudio realizado por Alccioly et al. (2000), evaluando fuentes de micronutrientes asociadas a tres concentraciones de calcáreo, se verificó que para las variables materia seca de la parte aérea y materia seca de la raiz (recolectadas 36 días después de la emergencia), valores de $24,26 \mathrm{~g}$ por planta y 9,80 g por planta, respectivamente, con aplicación de $4 \mathrm{t} \mathrm{ha}^{-1}$ de calcáreo y suministro de 3,6, $1,3,1,5$ y 4,0 $\mathrm{mg} \mathrm{dm}^{-3}$ de suelo de $\mathrm{Mn}, \mathrm{Cu}, \mathrm{Fe}$ y $\mathrm{Zn}$, 
respectivamente. Del mismo modo, Prado et al. (2008) evaluaron diferentes dosis de zinc y modos de aplicación, observándose diferencias significativas para materia seca de la parte aérea con relación al control, con un promedio de 32,1 g por planta, con un comportamiento similar para la materia seca de la raíz.

En cuanto a los componentes de producción de granos de maíz, no se observó ningún efecto del tratamiento para las variables número de hileras por mazorca (NFE), diámetro de la mazorca (DE), longitud de la mazorca (CE) y el masa de mil granos (MMG) (Tabla 3). Estos resultados están de acuerdo con los observados por Jamami et al. (2006) con respecto al NFE, DE y CE, al evaluar las dosis de Zn y B en el maíz. Sin embargo, se verificó efecto sobre los caracteres número de granos por mazorca (NGE) y el rendimiento de granos (PG). Para el NGE, se destacó el tratamiento de semillas líquido en la concentración de $600 \mathrm{~mL} \mathrm{ha}^{-1}$, obteniendo un promedio de 461,25 granos por mazorca, el mismo que presentó los mejores resultados en la MSPA e MSR, como se explicó anteriormente. Es de destacar que el NGE es uno de los principales componentes que determinan la productividad de los cultivos. En este sentido, Li et al. (2007) las concentraciones de micronutrientes en suelos o en el cultivo fueron fuertemente afectados por la disponibilidad del suelo de las concentraciones de potasio y fósforo, en un experimento a largo plazo.

Tabla 2. Materia seca de la parte aérea (MSPA) y la materia seca de raíz (MSR), a los 27 y 59 días después de la emergencia del maíz, promedio general y coeficiente de varianza $(\mathrm{CV})$ con relación a las fuentes y dosis de fertilizantes (Piracicaba, SP, 2009).

\begin{tabular}{|c|c|c|c|c|c|c|c|}
\hline \multirow{2}{*}{ Tratamientos } & \multirow{2}{*}{ Producto } & \multirow{2}{*}{ Dosis } & \multirow{2}{*}{$\begin{array}{c}\text { NPK } \\
(08-28-16)\end{array}$} & \multicolumn{2}{|c|}{$\operatorname{MSPA}\left(\mathrm{g} \mathrm{pl}^{-1}\right)$} & \multicolumn{2}{|c|}{$\operatorname{MSR}\left(\mathrm{g} \mathrm{pl}^{-1}\right)$} \\
\hline & & & & 27 días & 59 días & 27 días & 59 días \\
\hline 1 & Contr, $^{\#}$ & 0 & 0 & $28,8 \mathrm{~b}^{\$^{*}}$ & $301,9 \mathrm{~d}^{* *}$ & $1,52 \mathrm{~d}^{*}$ & $20,20 b^{* *}$ \\
\hline 2 & TS & 100 & 300 & $34,6 \mathrm{ab}$ & $662,9 \mathrm{abc}$ & $2,27 \mathrm{bc}$ & $20,72 \mathrm{~b}$ \\
\hline 3 & TS & 150 & 300 & $35,7 \mathrm{ab}$ & $779,0 \mathrm{ab}$ & $2,59 \mathrm{abc}$ & $43,87 \mathrm{a}$ \\
\hline 4 & TS & 200 & 300 & $35,1 \mathrm{ab}$ & $770,8 \mathrm{ab}$ & $2,22 \mathrm{bc}$ & $38,27 \mathrm{a}$ \\
\hline 5 & $\mathrm{TL}$ & 400 & 300 & $33,8 \mathrm{ab}$ & $655,6 \mathrm{bc}$ & $2,10 \mathrm{~cd}$ & $43,15 \mathrm{a}$ \\
\hline 6 & $\mathrm{TL}$ & 500 & 300 & $40,7 \mathrm{a}$ & $638,1 \mathrm{c}$ & $3,21 \mathrm{a}$ & $37,12 \mathrm{a}$ \\
\hline 7 & $\mathrm{TL}$ & 600 & 300 & $42,2 \mathrm{a}$ & $786,1 \mathrm{a}$ & $3,32 \mathrm{a}$ & $42,30 \mathrm{a}$ \\
\hline 8 & $\mathrm{~S}$ & 500 & 300 & $33,2 \mathrm{ab}$ & $673,9 a b c$ & $2,14 \mathrm{bcd}$ & $33,81 \mathrm{a}$ \\
\hline 9 & NPK & 0 & 600 & $38,3 \mathrm{ab}$ & $623,9 \mathrm{c}$ & $2,90 \mathrm{ab}$ & $38,05 \mathrm{a}$ \\
\hline Medio & & & & 35,82 & 654,71 & 2,47 & 35,27 \\
\hline CV (\%) & & & & 6,24 & 3,85 & 5,32 & 7,02 \\
\hline
\end{tabular}

$* *, * \mathrm{e}^{\mathrm{ns}}$ : significativo al 1 y $5 \%$ de probabilidad y no significativo por el test $\mathrm{F}$;

\# Contr: control; TS: tratamiento de semillas sólido; TL: tratamiento de semillas liquido; S: regulador del crecimiento y NPK (08/28/16) $300 \mathrm{~kg} \mathrm{ha}^{-1} .{ }^{\$}$ Promedio no unido por la misma letra en las columnas difieren en el nivel $5 \%$ de probabilidad de error por la prueba de Duncan.

Autores como Jamami et al. (2006) evaluando diferentes dosis de zinc en el suelo $\left(0,2\right.$ y $\left.4 \mathrm{~kg} \mathrm{ha}^{-1}\right)$, observaron respuestas positivas para la variable NGE. Estos mismos autores, destacando otros estudios, indican que esto se debe a este micronutrientes hacer parte de los sistemas enzimáticos, regulando el metabolismo de carbohidratos, fosfatos y proteínas además de la formación de auxinas, RNA y ribosomas, siendo esenciales en la regulación del crecimiento de la planta. También trabajando con $\mathrm{Zn}$ en dosis de 0,5 y $10 \mathrm{~kg} \mathrm{ha}^{-1}$, Gonçalves Júnior et al. (2007) no observaron respuestas a los componentes de rendimientos evaluados.

El rendimiento de grano fue incrementado (24\%) significativamente, solamente donde se aplicó el tratamiento de semillas líquido a una concentración de $600 \mathrm{~mL} \mathrm{ha}^{-1}\left(6949 \mathrm{~kg} \mathrm{ha}^{-1}\right)$ cuando comparado con el control (5601 kg ha ${ }^{-1}$ ) (Tabla 2). Los demás tratamientos tuvieron rendimientos intermedios y no significativos en comparación con el control. Se veri- ficó que los caracteres analizados con significación estadística, los comportamientos fueron similares, con más frecuencia ocurre la formación de tres grupos, donde el control tuvo los menores resultados, con los mejores resultados para el grupo que recibió el tratamiento semillas de líquido, especialmente en una concentración de $600 \mathrm{~mL} \mathrm{ha}^{-1}$. Lana et al. (2007) evaluaron cócteles de micronutrientes aplicados a través del suelo y las semillas en diferentes momentos, los cuales observaron un aumento en la productividad en relación con el control, lo que confirma la importancia de la utilización de micronutrientes en el cultivo del maíz. Otros autores como Souza et al. (1998), al evaluar la respuesta del maíz a la adición de dosis crecientes de $\mathrm{Zn}$, en el surco de la siembra, observaron que la adición de este micronutriente promovió incrementos significativos en el rendimiento de granos, sin embargo, no verificaron ventajas al aplicar dosis superiores a $5 \mathrm{~kg} \mathrm{ha}^{-1}$. Gonçalves Júnior et al. (2008), evaluando dosis de cobre 
$\left(0,5\right.$ y $\left.10 \mathrm{mg} \mathrm{dm}^{-3}\right)$ en el cultivo del maíz, no observaron respuesta.

Por otra parte, Fageria (2002), evaluando diferentes dosis de manganeso $(0,10,20,40,80,160$, 320 y $\left.640 \mathrm{mg} \mathrm{kg}^{-1}\right)$, observó aumento significativo de la productividad, siendo que el Mn aumentó también la absorción de $\mathrm{Mg}, \mathrm{Zn}$ y Fe por las plantas. Así como Hassegawa et al. (2008), indican que el uso de nitrógeno $\left(100 \mathrm{~kg} \mathrm{ha}^{-1}\right)$, zinc $\left(0 \mathrm{y} 1 \mathrm{~kg} \mathrm{ha}^{-1}\right)$ y boro $(0$ a $0,5 \mathrm{~kg} \mathrm{ha}^{-1}$ ) resultó en una reducción de los niveles de fumonisina B1 (FB1, FB2 y FB3) que son producidas naturalmente por el hongo Fusarium verticillioides. Autores como, Gupta et al. (2008) indican que la deficiencia de $\mathrm{Zn}$ provoca una alta sensibilidad en el cultivo del maíz, así como $\mathrm{Cu}$ Fe Mn po- seen una sensibilidad mediana y Mo y B poseen una sensibilidad baja. Por lo tanto, los tratamientos que contienen estos en mayor concentración, los micronutrientes para los que existe una mayor sensibilidad por el cultivo del maíz, poseen mejores resultados.

El regulador del crecimiento tuvo efecto sobre el cultivo del maíz, presentando un aumento de $13,6 \%$ en el rendimiento de grano $\left(6366 \mathrm{~kg} \mathrm{ha}^{-1}\right)$, lo que demuestra haber efecto hormonal en el cultivo del maíz, aunque una sola dosis haya sido probada. La aplicación del doble de la dosis del fertilizante NPK no mostró mejora en el rendimiento de grano $\left(6770 \mathrm{~kg} \mathrm{ha}^{-1}\right)$ y en las demás características evaluadas, estando en una posición intermedia entre el mejor y el peor tratamiento (Tablas 2 y 3 ).

Tabla 3. Número de granos por mazorca (NGE), número de hileras por mazorca (NFE), diámetro de mazorcas (DE, $\mathrm{cm}$ ), longitud de la mazorca $(\mathrm{CE}, \mathrm{cm})$, masa de mil granos $(\mathrm{MMG})$, rendimiento de granos $\left(\mathrm{PG}, \mathrm{kg} \mathrm{ha}^{-1}\right)$, porcentaje de incremento en la producción de granos con relación al control (A\%) de maíz, promedio general y el coeficiente de variación, con relación a las fuentes y dosis de fertilizantes (Piracicaba, SP, 2009).

\begin{tabular}{ccccccccccc}
\hline Trat. & Produto & Dose & NPK & NGE & NFE & DE & CE & MMG & PG & $\%$ A \\
\hline 1 & Contr,\# & 0 & 0 & $386,3 \mathrm{~b}^{* \$}$ & $14,0^{\text {ns }}$ & $4,8^{\text {ns }}$ & $\begin{array}{c}13,8 \\
\text { ns }\end{array}$ & $348,4^{\text {ns }}$ & $5601 \mathrm{~b}^{* *}$ & -- \\
2 & TS & 100 & 300 & $408,8 \mathrm{ab}$ & 13,7 & 4,8 & 15,1 & 381,7 & $6744 \mathrm{ab}$ & 20,4 \\
3 & TS & 150 & 300 & $425,5 \mathrm{ab}$ & 13,8 & 4,8 & 15,1 & 372,3 & $6508 \mathrm{ab}$ & 16,2 \\
4 & TS & 200 & 300 & $417,5 \mathrm{ab}$ & 13,6 & 4,8 & 14,5 & 363,5 & $6440 \mathrm{ab}$ & 14,9 \\
5 & TL & 400 & 300 & $408,5 \mathrm{ab}$ & 13,6 & 4,8 & 14,4 & 355,6 & $6532 \mathrm{ab}$ & 16,6 \\
6 & TL & 500 & 300 & $405,5 \mathrm{ab}$ & 13,5 & 4,8 & 15,0 & 370,5 & $6880 \mathrm{ab}$ & 22,8 \\
7 & TL & 600 & 300 & $461,3 \mathrm{a}$ & 13,8 & 4,9 & 15,6 & 359,2 & $6949 \mathrm{a}$ & 24,0 \\
8 & $\mathrm{~S}$ & 500 & 300 & $390,8 \mathrm{ab}$ & 13,4 & 4,9 & 15,3 & 384,9 & $6366 \mathrm{ab}$ & 13,6 \\
9 & NPK & - & 600 & $410,0 \mathrm{ab}$ & 13,7 & 4,8 & 14,7 & 360,2 & $6770 \mathrm{ab}$ & 20,8 \\
\hline Média & & & & 412,7 & 13,7 & 4,8 & 14,8 & 366,3 & 6532 & \\
CV (\%) & & & & 3,67 & 1,41 & 1,00 & 3,21 & 2,32 & 4,43 & \\
\hline
\end{tabular}

$* *, * \mathrm{e}^{\mathrm{ns}}$ : significativo al 1 y $5 \%$ de probabilidad y no significativo por el test $\mathrm{F}$;

\# Contr: control; TS: tratamiento de semillas sólido; TL: tratamiento de semillas liquido; S: regulador del crecimiento y NPK (08/28/16) $300 \mathrm{~kg} \mathrm{ha}^{-1}$. \$ Promedio no unido por la misma letra en las columnas difieren en el nivel $5 \%$ de probabilidad de error por la prueba de Duncan.

El porcentaje de aumento de la productividad en general fue expresiva, variando de 13,6 a $24 \%$ (Tabla 3). Reis Junior (2004) evaluando la fertilización foliar con $\mathrm{B}, \mathrm{Cu}, \mathrm{Mn}, \mathrm{Zn}$ y mezclas de los mismos, en Chapadão do Sul MS, observó aumento de la productividad que oscila desde $9,5-16,1 \%$ para B, $4-12,5 \%$ para $\mathrm{Cu}, 6,8-21,6 \%$ para $\mathrm{Mn}, 6,1-12,7 \%$ para $\mathrm{Zn}$ y $3,8-9,5 \%$ para la mezcla de micronutrientes. Es importante observar también que la interacción entre los nutrientes es uno de los factores que pueden interferir en el rendimiento de los cultivos, pudiendo ser positivo o negativo (FAGERIA; BALIGAR, 1997). Un ejemplo de esto es la interacción de $\mathrm{Cu}$ y $\mathrm{Mn}$ con $\mathrm{N}$, cuyo uso de dosis crecientes de $\mathrm{N}$ promovió aumento en la disponibilidad de estos micronutrientes (FERREIRA et al., 2001). Además, las concentraciones de nutrientes en el suelo y las condiciones climáticas pueden influir en la interacción y, por consiguiente, en respuesta a la fertilización.

\section{CONCLUSIÓN}

El tratamiento de semillas, ya sea en forma sólida o líquida, promueve un aumento en la acumulación de materia seca en la parte aérea y raíces, así como en el número de granos y en el rendimiento de granos de maíz, siendo la dosis de $600 \mathrm{~mL} \mathrm{ha}^{-1}$ de la que promueve las mejores productividades.

\section{REFERENCIAS}

ACCIOLY, A. M. A. et al. Pó de forno elétrico de siderurgia como fonte de micronutrientes e de contaminantes para plantas de milho. Pesquisa Agropecuária Brasileira, Brasília, v. 35, n. 7, p. 1483-1491, 2000.

AMARAL FILHO, J. P. R. et al. Espaçamento, densidade populacional e adubação nitrogenada na cul- 
tura do milho. Revista Brasileira de Ciência do Solo, Viçosa, v. 29, n.3, p.467-473, 2005.

BATAGLIA, O. C. Análise química de plantas. In: FERREIRA, M.E.; CRUZ, M.C.P. (Ed.) Micronutrientes na agricultura. Piracicaba: Associação Brasileira para a Pesquisa da Potassa e do Fosfato, p. 289-308, 1991.

CAIRES, E. F.; FONSECA, A. F. Absorção de nutrientes pela soja cultivada no sistema de plantio direto em função da calagem na superfície. Bragantia, Campinas, v. 59, n. 2, p. 213-220, 2000.

CONAB. Companhia Nacional de Abastecimento. Levantamento da safra de 2013/2014, 2014. Disponível em: <http://www.conab.gov.br/> Acesso em: 02 fev. 2014

CRUZ, S. C. S. et al. Adubação nitrogenada para o milho cultivado em sistema plantio direto, no Estado de Alagoas. Revista Brasileira de Engenharia Agrícola e Ambiental, Viçosa, v. 12, n. 1, p. 62-68, 2008.

EMBRAPA. Centro Nacional de Pesquisa de Solos. Sistema Brasileiro de Classificação de Solos. 3 ed. Rio de Janeiro, RJ, 2013, p.353.

ERNANI, P. R.; BAYER, C.; MAESTRI, L. Corn yield as affected by liming and tillage system on an acid Brazilian Oxisol. Agronomy Journal, Madison, v. 94, n. 2, p. 305-309, 2002.

FAGERIA, N. K. Influence of micronutrients on dry matter yield and interaction with other nutrients in annual crops. Pesquisa Agropecuária Brasileira, Brasília, v. 37, n. 12, p. 1765-1772, 2002.

FAGERIA, N. K.; BALIGAR, V. C. Response of common bean, upland rice, corn, wheat and soybean to fertility of an Oxisol. Journal of Plant Nutrition, Wenheim, v. 20, n. 10, p. 1279-1289, 1997.

FANCELli, A. L.; DOURADO NETO, D. Agropecuária: A produção de milho. 2. Ed. Guaiba: Livroceres, 2000, p.360.

FERREIRA, A. C. B. et al. Características agronômicas e nutricionais do milho adubado com nitrogênio, molibdênio e zinco. Sciencia Agricola, Viçosa, v. 58 , n. 1, p. 131-138, 2001

GONÇALVES JÚNIOR, A. C. et al. Produtividade e componentes de produção do milho adubado com $\mathrm{Cu}$ e NPK em um Argissolo. Scientia Agricola, Piracicaba, v. 9, n. 1, p. 35-40, 2008.

GONÇALVES JÚNIOR, A. C. et al. Produtividade do milho em resposta a adubação com NPK e Zn em
Argissolo Vermelho-amarelo Eutrófico e Latossolo Vermelho Eutroférrico. Ciência Agrotécnica, Lavras, v. 31, n. 4, p. 1231-1236, 2007.

GUPTA, U. C.; KENING, W.; LIANG, S. Micronutrients in soils, crops, and livestock. Earth Science Frontiers, Beijing, v. 15, n. 5, p. 110-125, 2008.

HASSEGAWA, R. H. et al. Influence of macro-and micronutrient fertilization on fungal contamination and fumonisin production in corn grains. Food Control, Amsterdam, v. 19, n.2, p.36-43, 2008.

JAMAMI, N. et al. Resposta da cultura do milho (Zea mays L.) à aplicação de boro e de zinco no solo. Acta Scientiarum, Agronomy, Maringá, v. 28, n. 1, p. $99-105,2006$.

LANA, A. M. Q. et al. Doses, fontes e épocas de aplicação de micronutrientes na cultura do milho. Magistra, Cruz das Almas, v. 19, n. 1, p. 76-81, 2007.

LEITE, U. T. et al. Níveis críticos de boro, cobre, manganês e zinco em milho. Bioscience Journal, Uberlândia, v. 19, n. 2, p. 115-125, 2003.

LI, B.Y. et al. Soil micronutrient availability to crops as affected by long-term inorganic and organic fertilizer applications. Soil \& Tillage Research, Amsterdam, v. 96, n. 1-2 , p.166-173, 2007.

MARTIN, T. N. et al. Perfil do manejo da cultura de milho no sudoeste do Paraná. Revista Ceres, Viçosa, v. 58, n. 1, p. $1-8,2011$

PRADO, R. M. et al. Modos de aplicação de zinco na nutrição e na produção de matéria seca do milho BRS 1001. Bioscience Journal, Uberlandia, v. 24, n. 1, p. 67-74, 2008.

RAIJ, B. V. et al. Recomendações de adubação e calagem para o Estado de São Paulo, 2.ed. Campinas: Instituto Agronômico/Fundação IAC, (Boletim Técnico, 100), 1997, p.285.

REIS JUNIOR, R. A. Resultados de pesquisa em fertilidade do solo e nutrição mineral de plantas safra 2002/2003, Informativo da Fundação Chapadão, Chapadão do Sul, 2004, p.5.

SAULNIER, L. Les grains de céréales: diversité et compositions nutritionnelles Cereals grains: diversity and nutritional composition. Cahiers de nutrition et diététique, Paris, v. 47, n.1, p. 4-15, 2012.

SOUZA, E. C. A. et al. Resposta do milho a adubação com fósforo e Zn. Pesquisa Agropecuária Brasileira, Brasília, v. 33, n. 7, p. 1031-1036, 1998.

TOMAZELA, A. L. et al. Doses de nitrogênio e fon- 
tes de $\mathrm{Cu}$ e $\mathrm{Mn}$ suplementar sobre a severidade da ferrugem e atributos morfológicos do milho. Revista Brasileira de Milho e Sorgo, Sete Lagoas, v. 5, n. 2, p. 192-201, 2006.

YAMADA, T. Deficiências de micronutrientes, ocorrência, detecção e correção: o sucesso da experiência brasileira, Informações Agronômicas 105, 2004, p.12. 